\title{
In Situ Observation of Vapor-Assisted 2D-3D Heterostructure Formation for Stable and Efficient Perovskite Solar Cells
}

Zhou Liu,${ }^{\dagger}$ Ke Meng, ${ }^{\dagger}$ Xiao Wang, ${ }^{\dagger}$ Zhi Qiao,${ }^{\dagger}$ Qiaofei Xu,${ }^{\dagger}$ Shunde Li,${ }^{\dagger}$ Lei Cheng,,${ }^{\dagger}$

Zhimin $\mathrm{Li}^{\dagger},+\star$ and Gang Chen ${ }^{*}, \dagger, \S$

'School of Physical Science and Technology, ShanghaiTech University, Shanghai 201210,

China

${ }^{\ddagger}$ School of Environment and Architecture, University of Shanghai for Science and

Technology, Shanghai 200093, China

${ }^{\S}$ Shanghai Synchrotron Radiation Facility, Shanghai Institute of Applied Physics, Chinese

Academy of Sciences, Shanghai 201204, China

*e-mail: gchen@shanghaitech.edu.cn 


\section{Supporting Figures}

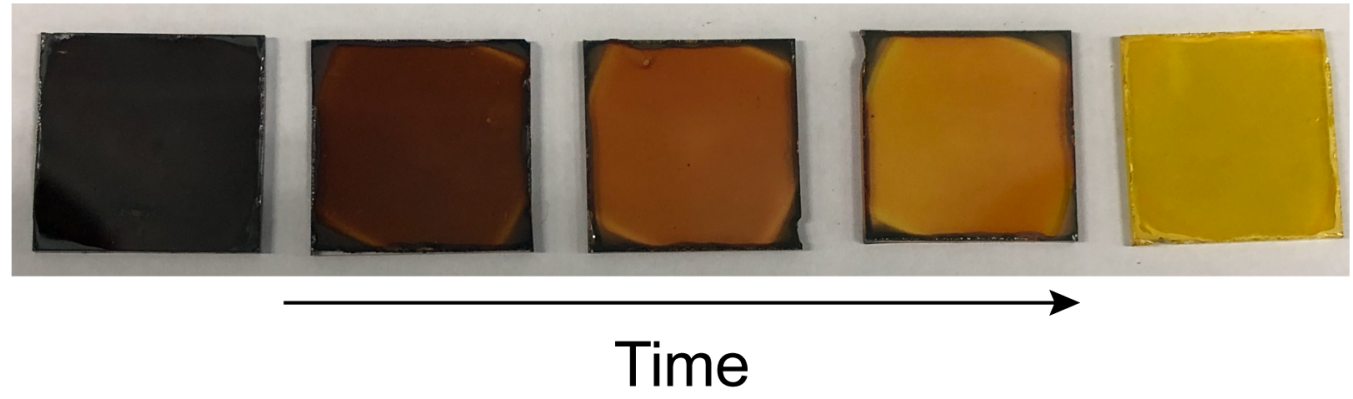

Figure S1. Digital photographs of the BAV treated perovskite films for different exposure times.
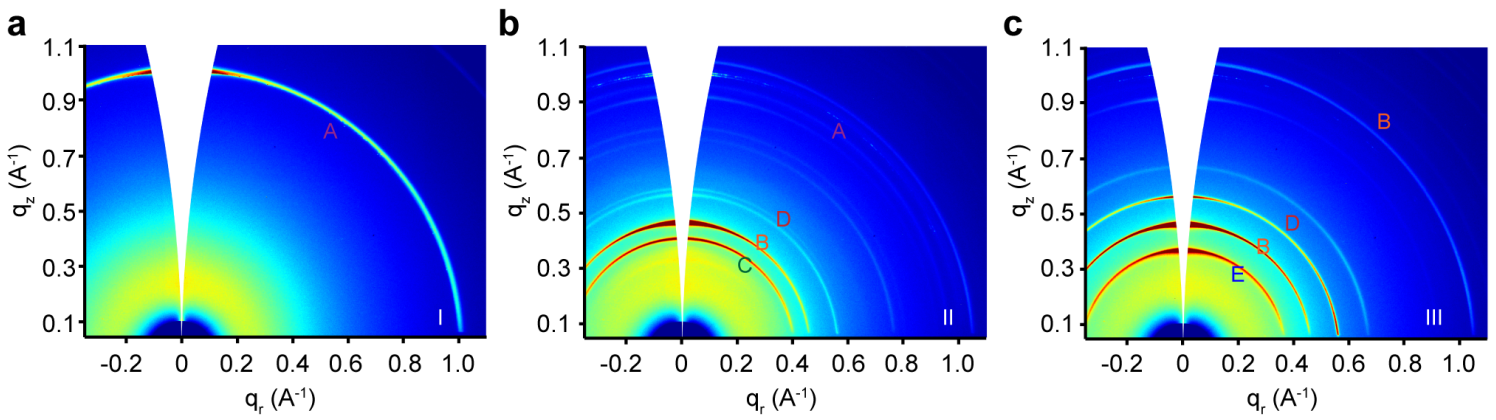

Figure S2. Typical 2D GI-XRD patterns of the sample taken during the $3 \mathrm{D}$ to $2 \mathrm{D}$ transition at the points of (a) I, (b) II and (c) III as denoted in Figure 1a of the main text. 

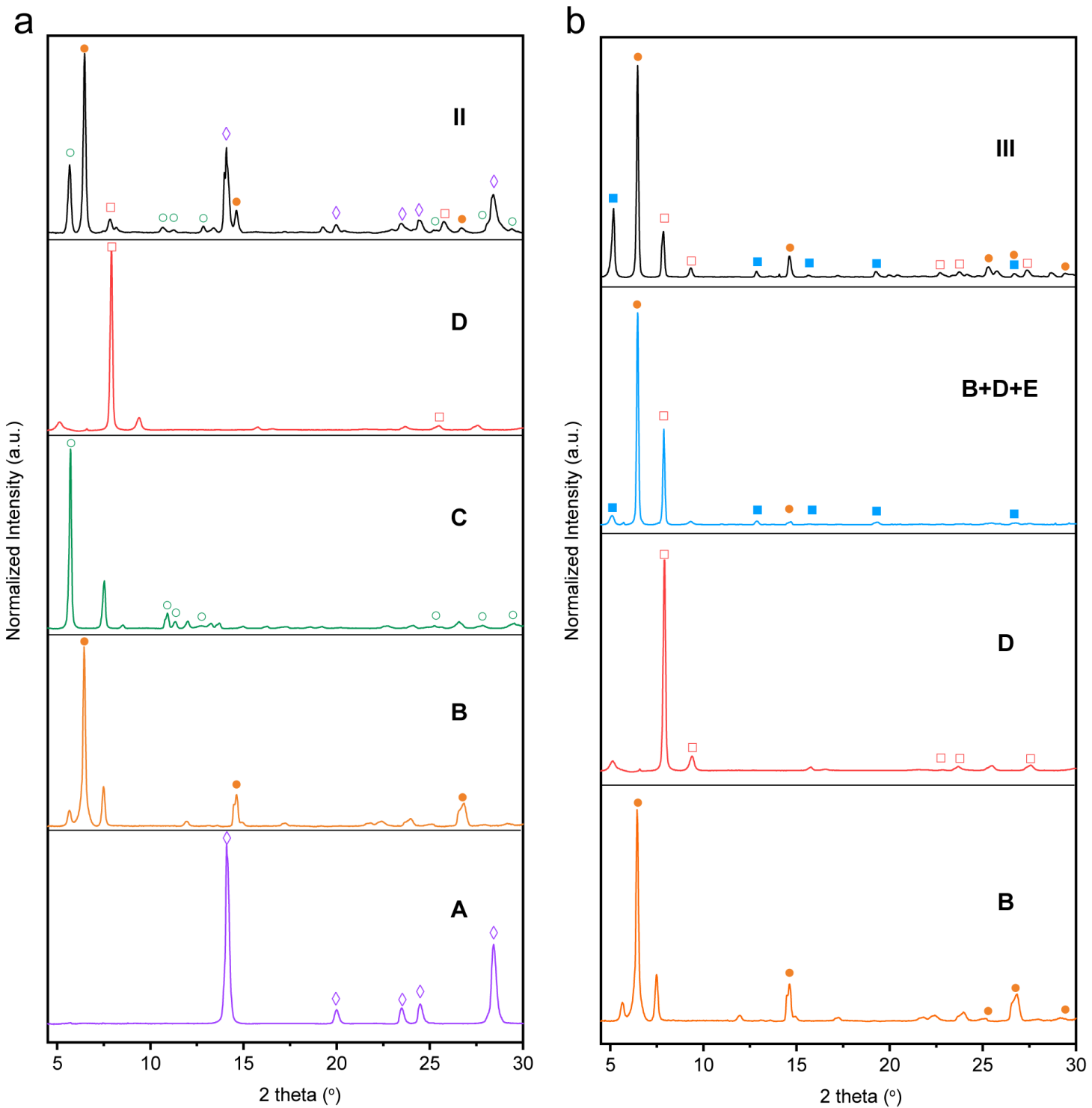

Figure S3. The XRD patterns taken at points II and III (denoted in Figure 1a) are compared with those of species A, B, C, D and E. The purple rhombus, orange solid circle, green hollow circle, red hollow square and blue solid square represent the typical diffraction peaks of species A, B, C, D and E, respectively. 
a

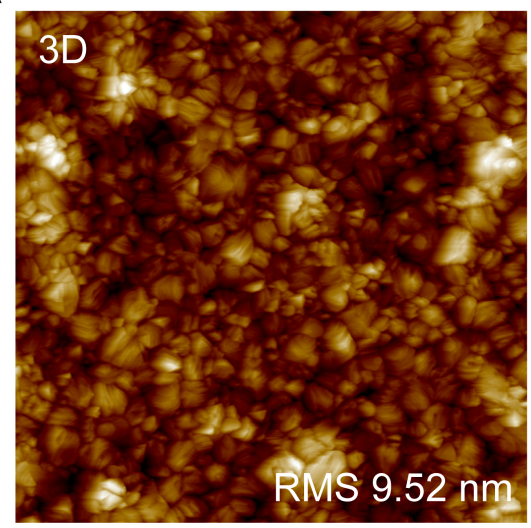

b

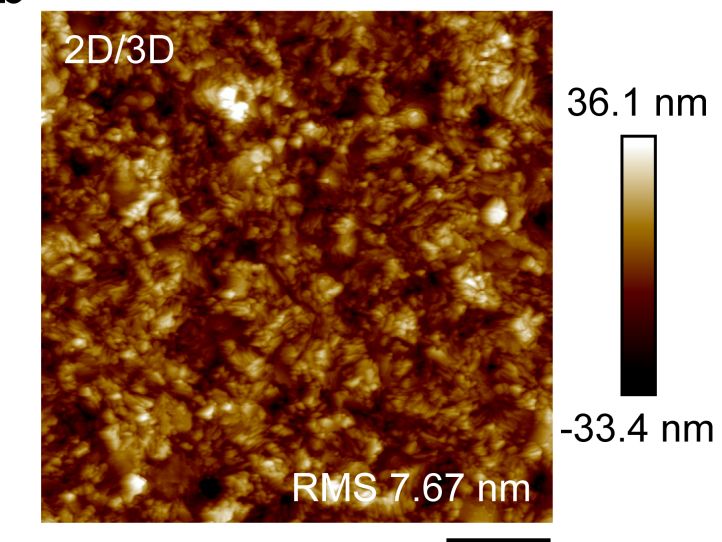

$.1 \mathrm{~nm}$

Figure S4. The AFM images of $3 \mathrm{D}$ and $2 \mathrm{D} / 3 \mathrm{D}$ perovskite films. The scale bar is $1 \mu \mathrm{m}$.

a

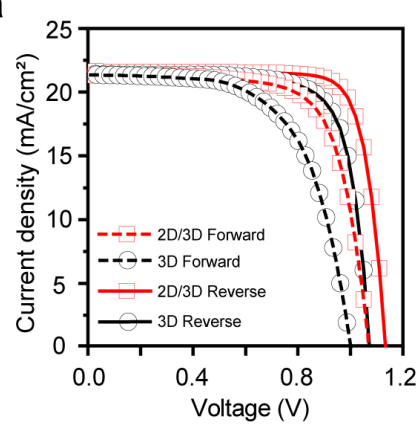

$46.6 \mathrm{~nm}$

$-39.5 \mathrm{~nm}$

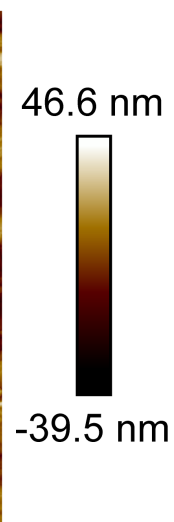

b
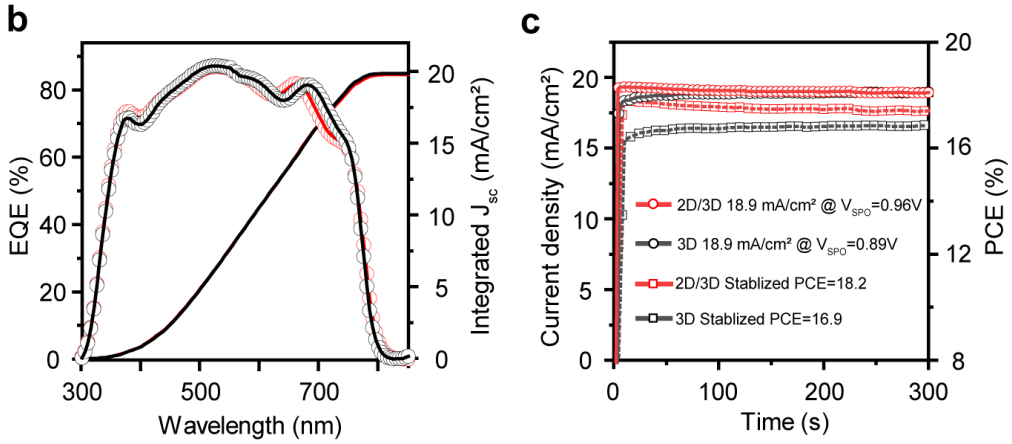

Figure S5. (a) J-V curves, (b) EQE, and (c) stabilized power output (SPO) of the typical PSC devices based the $3 \mathrm{D}$ and $2 \mathrm{D} / 3 \mathrm{D}$ heterostructured perovskite films. 


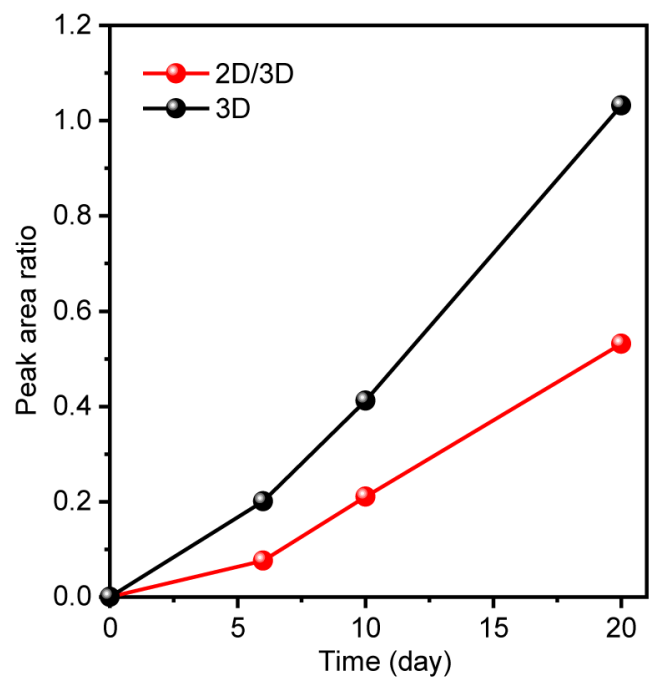

Figure S6. The peak area ratios between the $\mathrm{PbI}_{2}(002)$ peak and the perovskite (110) peak for the 3D (black line) and 2D/3D (red line) perovskite films as a function of aging time at $85^{\circ} \mathrm{C}$ in $\mathrm{N}_{2}$ atmosphere. 


\section{Supporting Tables}

Table S1. Summary of the photovoltaic parameters of the devices based on the 2D/3D perovskite films with the BAV treatment for various durations.

\begin{tabular}{|c|c|c|c|c|c|}
\hline Samples & & $\mathrm{V}_{\mathrm{oc}}(\mathrm{V})$ & $\mathrm{J}_{\mathrm{sc}}\left(\mathrm{mA} / \mathrm{cm}^{2}\right)$ & $\mathrm{FF}(\%)$ & PCE (\%) \\
\hline \multirow{2}{*}{ Control } & average & 1.10 & 20.50 & 77.54 & 17.43 \\
\hline & $\max$ & 1.14 & 20.41 & 81.78 & 18.98 \\
\hline \multirow{2}{*}{$30 \mathrm{~s}$ treatment } & average & 1.11 & 21.15 & 78.22 & 18.37 \\
\hline & $\max$ & 1.14 & 21.00 & 80.7 & 19.02 \\
\hline \multirow{2}{*}{$1 \mathrm{~min}$ treatment } & average & 1.13 & 21.34 & 78.85 & 18.63 \\
\hline & $\max$ & 1.16 & 20.89 & 80.4 & 19.48 \\
\hline \multirow{2}{*}{3 min treatment } & average & 1.14 & 18.69 & 78.64 & 16.90 \\
\hline & $\max$ & 1.16 & 19.18 & 80.4 & 18.05 \\
\hline \multirow{2}{*}{5 min treatment } & average & 1.10 & 18.05 & 72.26 & 14.35 \\
\hline & $\max$ & 1.11 & 19.2 & 73.2 & 15.6 \\
\hline \multirow{2}{*}{30 min treatment } & average & 0.44 & 0.58 & 46.4 & 0.12 \\
\hline & $\max$ & 0.42 & 0.65 & 54.7 & 0.15 \\
\hline
\end{tabular}

Table S2. The parameters obtained from fitting the time-resolved PL spectra of the 3D and 2D/3D heterostructured perovskite films. $\tau_{1}$ and $\tau_{2}$ are the lifetimes of the fast and slow decay processes, $\tau_{\text {avg }}$ is the average lifetime, $A_{1}$ and $A_{2}$ are two fitting constants.

\begin{tabular}{|c|c|c|c|c|c|}
\hline Pamples & $\tau_{1}(\mathrm{~ns})$ & $\tau_{2}(\mathrm{~ns})$ & $\tau_{\text {avg }}(\mathrm{ns})$ & $A_{1}$ & $A_{2}$ \\
\hline 3D & 71.6 & 286.3 & 129.0 & 4230 & 13929 \\
\hline 2D/3D & 79.9 & 319.7 & 165.5 & 2602 & 11690 \\
\hline
\end{tabular}


Table S3. The parameters obtained from fitting the EIS spectra for the PSC devices based on the 3D and $2 \mathrm{D} / 3 \mathrm{D}$ heterostructured perovskite films, the sample area in square centimeter.

\begin{tabular}{|c|c|c|c|c|c|}
\hline Samples $\quad$ Parameters & $\mathrm{R}_{\mathrm{ct}}(\Omega)$ & $\mathrm{R}_{\mathrm{rec}}(\Omega)$ & $\mathrm{R}_{\mathrm{s}}(\Omega)$ & $\mathrm{C}_{\mathrm{ct}}(\mathrm{F})$ & $\mathrm{C}_{\mathrm{rec}}(\mathrm{F})$ \\
\hline $3 \mathrm{D}$ & 96.4 & $5.6 \times 10^{6}$ & 5.4 & $2.1 \times 10^{-8}$ & $4.3 \times 10^{-7}$ \\
\hline $2 \mathrm{D} / 3 \mathrm{D}$ & 100.0 & $8 \times 10^{6}$ & 2.8 & $2.4 \times 10^{-8}$ & $6.7 \times 10^{-7}$ \\
\hline
\end{tabular}

